\title{
Retinal ganglion cell survival after severe optic nerve injury is modulated by crosstalk between JAK/STAT signaling and innate immune responses in the zebrafish retina
}

Si Chen ${ }^{1,2,3}$, Kira L. Lathrop ${ }^{2,4}$, Takaaki Kuwajima ${ }^{2,5 *}$, Jeffrey M. Gross ${ }^{2,5 *}$

1. Eye Center of Xiangya Hospital, Central South University, 410008, Changsha, Hunan, P.R. China,

2. Department of Ophthalmology, The University of Pittsburgh School of Medicine, Pittsburgh, PA 15213, USA,

3. Hunan Key Laboratory of Ophthalmology, 410008, Changsha, Hunan, P.R. China,

4. Department of Bioengineering, University of Pittsburgh Swanson School of Engineering, Pittsburgh, Pennsylvania, United States of America.

5. Department of Developmental Biology, Louis J. Fox Center for Vision Restoration, The University of Pittsburgh School of Medicine, Pittsburgh, PA 15213, USA,

Authors for correspondence (grossjm@pitt.edu; kuwajima@pitt.edu) 


5

\section{ABSTRACT:}

Visual information is transmitted from the eye to the brain along the optic nerve, a structure composed of retinal ganglion cell (RGC) axons. The optic nerve is highly vulnerable to damage in neurodegenerative diseases like glaucoma and there are currently no FDA-approved drugs or therapies to protect RGCs from death. Zebrafish possess remarkable neuroprotective and regenerative abilities and here, utilizing an optic nerve transection (ONT) injury and an RNA-seq-based approach, we identify genes and pathways active in RGCs that may modulate their survival. Through pharmacological perturbation, we demonstrate that JAK/STAT pathway activity is required for RGC survival after ONT. Furthermore, we show that immune responses directly contribute to RGC death after ONT; macrophages/microglia are recruited to the retina and blocking neuroinflammation or depleting these cells after ONT rescues survival of RGCs. Taken together, our results support a model in which pro-survival signals in RGCs, mediated by JAK/STAT signaling, counteract the activity of innate immune responses to modulate RGC vulnerability and resilience in the zebrafish retina after severe optic nerve damage.

87


4

\section{INTRODUCTION:}

Visual information is transmitted from the eye to the brain along the optic nerve $(\mathrm{ON})$, a structure composed of retinal ganglion cell (RGC) axons. The $\mathrm{ON}$ is highly vulnerable to damage and is compromised after acute injury and in neurodegenerative diseases such as glaucoma. In glaucoma, RGC axons are the initial site of injury; this causes the RGCs to die and ultimately results in irreversible loss of visual function. Neuroprotective strategies for glaucoma treatment seek to maintain the health of RGCs even after axons have been damaged, or to prevent initial damage to the RGC axon itself (Almasieh et al., 2012; Chang and Goldberg, 2012). There has been substantial progress in identifying the molecular and cellular events that lead to RGC death in the glaucomatous eye (Almasieh et al., 2012; Chang and Goldberg, 2012; Syc-Mazurek and Libby, 2019); however, no FDA-approved therapies currently exist to protect RGCs from death. This highlights the critical need for new neuroprotective strategies that preserve RGCs during glaucoma or after acute ocular trauma.

5
example, in mouse, $\sim 65 \%$ of RGCs are lost within 7 days of optic nerve injury (ONI), and $>90 \%$ by 28 days ( $\mathrm{Li}$ et al., 2020). Mammals are also unable to regenerate RGCs after ONI, leading to irreparable vision loss. Unlike mammals, zebrafish possess remarkable neuroprotective and regenerative capacity in the central nervous system (Cigliola et al., 2020; Lahne et al., 2020). When the ON is damaged by crush or transection, zebrafish mount a robust regenerative response and regenerate RGC axons, restoring visual connections and function (Diekmann et al., 2015; Dhara et al., 2019). Moreover, it has 
83 been reported that $\sim 75 \%$ of zebrafish RGCs are protected from death after ONI, even to

84 7-weeks post-injury (Zou et al., 2013), but the mechanisms underlying neuroprotection

85 are unknown. With an interest in developing novel strategies to preserve RGCs during

86 glaucoma and other trauma, here, we identify potential neuroprotective factors/pathways

87 in zebrafish that mediate RGC survival after ONI.

88 


\section{MATERIALS AND METHODS:}

\section{Animals}

Zebrafish (Danio rerio) in this study were 3-5 months old with an equal number of males and females used in all experiments. Transgenic lines used are is/2b:GFP (Pittman et al., 2008) and mpeg1:mCherry (Ellett et al., 2011); a gift from Dr. Neil Hukriede, University of Pittsburgh. Animals were maintained under standard conditions at $28.5 \mathrm{C}$ on a $14 \mathrm{~h}$ light/10 $\mathrm{h}$ dark cycle. There were no differences in outcomes based on gender of the fish and therefore all data were combined for analyses. All animals were treated in accordance with provisions established by the University of Pittsburgh School of Medicine Institutional Animal Care and Use Committee. Biological replicates (Ns) are provided in Figure legends for each experiment. At least three independent biological replicates were used per experiment.

\section{Optic nerve transection}

Optic nerve transection (ONT) was performed as previously described (Elsaeidi et al., 2014; Zou et al., 2013). Zebrafish were anesthetized in 0.03\% tricaine buffer (MS-222; Fisher Scientific) and placed on a moist tissue paper under a dissecting scope (Leica E65S). ONT surgery was performed on the left eye. After removal of the connective tissue, the eyeball was pulled out from the orbit gently using forceps. The ON and ophthalmic artery that runs along with the $\mathrm{ON}$ were exposed and the $\mathrm{ON}$ was then completely transected with another forcep, after which the eye was placed back in the orbit. Any animals where bleeding was observed were euthanized and not used for analysis. The right eye was subjected to a sham surgery as control: connective tissue 
was removed, the eye was pulled out from the orbit gently, and then placed back in the orbit. Fish were returned to system water in separate tanks to recover.

\section{RGC isolation and fluorescence-activated cell sorting (FACS)}

Retinae were harvested from is/2b:GFP zebrafish at 12 and 24 hours post-injury (hpi) in biological triplicate. Four retinae were collected per sample. For retinal isolations and cell dissociation, animals were euthanized by tricaine overdose and transferred to PBS for enucleation. To achieve single cell suspension, the eyeball was rinsed in 1X PBS postenucleation and incubated in StemPro ${ }^{\mathrm{TM}}$ Accutaset $^{\mathrm{TM}}$ Cell Dissociation Reagent (Thermo Fisher, \#A1110501) at 28.5 C for 40min in a water bath. The cell suspension was then passed through a $70 \mu \mathrm{m}$ cell strainer (Fisher Scientific) and gently pelleted by centrifugation at 4500rpm for $5 \mathrm{~min}$ at $4 \mathrm{C}$. After two washes in ice cold $1 \mathrm{X}$ PBS, cells were resuspended in ice cold $5 \%$ FBS in 1 X PBS. GFP ${ }^{+}$cells were sorted using a FACS Aria llu cell sorter (BD Biosciences) at the Flow Cytometry Core at the University of Pittsburgh School of Medicine Department of Pediatrics. The gate for FACS was set by GFP intensity for both injured (ONT) and intact (control) is/2b:GFP samples. The same gating settings were used for both the ONT and control RGC samples and for all biological replicates.

\section{RNA-seq and bioinformatics analyses}

Library preparation, quality control analysis, and next generation sequencing were performed by the Health Sciences Sequencing Core at Children's Hospital of Pittsburgh as previously described (Leach et al., 2020). cDNA sequencing libraries were prepared 
using a SmartSeq HT kit (Takara Bio) and Illumina Nextera XT kit (Illumina Inc.). 2 X 75 paired-end, 150 cycle sequencing was performed on a NextSeq 500 system (Illumina Inc.), aiming for 40 million reads per sample. Raw read and processed data files are available in GEO: GSE171426. After sequencing, raw read data were imported to the CLC Genomics Workbench (Qiagen Digital Insights) licensed through the Molecular Biology Information Service of the Health Sciences Library System at the University of Pittsburgh. After mapping trimmed reads to the Danio rerio reference genome (assembly GRCz11), differentially expressed genes (DEG) from the 24hpi time point were identified using the following filter: the maximum of the average group RPKM value $>1.5$, absolute fold change $>2$, false discovery rate $(F D R) p$-value $<0.05$. Genes with TPM=0 in one or more replicates were excluded. This filtering strategy was also used for DEG at $12 \mathrm{hpi}$ and a second analysis was performed where the FDR $p$-value $<0.05$ was switched to a $p$ value $<0.05$. Pathway enrichment analyses were performed using DAVID Bioinformatics Resources 6.8 (https://david.ncifcrf.gov) and using the KEGG database.

\section{Pharmacological experiments}

To assess toxicity and efficacy, the JAK inhibitor, Pyridone 6 (P6), or dexamethasone (both Sigma-Aldrich) was intravitreally (IV) injected into the intact and injured retina at several different concentrations, as previously described (Elsaeidi et al., 2014), and RGC survival was quantified. Based on (Elsaeidi et al. 2014; Bollaerts et al. 2019), 5uM P6 and 10uM dexamethasone were utilized. The first dose of each compound $(2 \mu \mathrm{L})$ was injected immediately after ONT (0dpi) and the second dose $(2 \mu \mathrm{L})$ was injected at $1 \mathrm{dpi}$. $2 \mu \mathrm{l}$ of $0.05 \%$ DMSO was injected for all control doses. To deplete macrophages/microglia, fish 
were immersed in 500nM PLX3397 (Fisher Scientific) in system water, as previously described (Kanagaraj et al., 2020). PLX3397 exposure started 1 day before ONT and system water containing PLX3397 was replaced daily during the experiment.

\section{Immunohistochemistry}

Immunofluorescence staining on retinal cryosections and flat-mounted retinae were performed as previously described (Uribe and Gross, 2007; Zou et al., 2013) with the addition of an antigen retrieval step consisting of $100 \%$ methanol incubation at $-20 \mathrm{C}$ for 30 minutes for staining pSTAT3 (MBL International Corporation, D128-3). For retinal flatmounts, after euthanasia, fish were decapitated and heads were fixed in $4 \%$ paraformaldehyde (PFA) at 4C overnight. The retina was dissected in ice cold PBS, washed in $0.1 \%$ PBST (Triton X-100 in PBS), and then incubated in a $1.5 \mathrm{ml}$ tube on a rotator overnight at 4C with 4C4 (1:200, a kind gift of Dr. Peter Hitchcock, University of Michigan School of Medicine; Craig et al., 2008), pSTAT3 (1:100, MBL), cleaved caspase 3 (1:200, Abcam, ab13847) and mCherry (1:200, Takara Bio USA Inc./Clontech Laboratories, 632543). Retinae were then washed in $0.1 \%$ PBST for $3 \times 10$ minutes at room temperature and incubated with goat-anti mouse Cy3 (1:250, Jackson ImmunoResearch Labs, 115-165-166) or goat-anti rabbit Alexa 647 (1:500, Cell Signaling Technology, 8940) secondary antibody for 3 hours at room temperature. Samples were then washed with $0.1 \%$ PBST for $3 \times 10$ minutes and carefully cut into 4 quadrants and mounted on slides with DAPI Vectashield (Vector Laboratories, H-1200). For cryosections, samples were prepared as previously described (Uribe and Gross, 2007); 
zn-8 (Zebrafish International Resource Center) was used at a 1:200 dilution and all other antibodies were used at the same concentrations as for the retinal flat-mount.

\section{BrdU Incorporation assays}

Adult is/2b:GFP+ fish were immersed in 10mM BrdU (Sigma Aldrich) dissolved in system water for from $6 \mathrm{dpi}$ to $7 \mathrm{dpi}$, and sacrificed at $7 \mathrm{dpi}$. As a positive control for BrdU incorporation and immunohistochemistry, a needle poke injury was performed after (Fausett and Goldman, 2006) and fish were exposed to BrdU for 24 hours prior to being sacrificed. Immunohistochemistry for BrdU proceeded as described above for other antibodies, with the addition of a $10 \mathrm{~min}$ incubation of $4 \mathrm{~N} \mathrm{HCl}$ at $37 \mathrm{C}$ to relax chromatin. anti-BrdU (Abcam, ab6326) was used at 1:200 dilution.

\section{Confocal microscopy, image processing and quantification}

For is/2b:GFP imaging, retinal flat-mounts were prepared as above, with the head fixed in $4 \%$ PFA overnight at $4 \mathrm{C}$ and the retina dissected and mounted on the second day. Images were taken using Olympus Fluoview FV1200 laser scanning microscope (Olympus Corporation). Images were taken from each of the 4 quadrants (1 peripheral, 1 central per quadrant) at 40X magnification. Quantification of RGC numbers was performed using particle analysis in ImageJ after setting up a consistent threshold for all images. RGC survival was calculated as the ratio of is/2b:GFP ${ }^{+}$RGCs in the left (ONT+) eye/ is/2b:GFP+ RGCs in the right (ONT- control) eye of the same fish. 
Quantification of macrophages/microglia was performed using Imaris 9.6.0

204 (Bitplane). Confocal images were first converted into Imaris files, 3D rendered surfaces

205

206

207

208

209

210

211

212

213

214

215

216

217

218

219

220

221

222

223

224

225

were then created for mCherry or $4 \mathrm{C} 4$ using the same algorithm (smoothing $=0.4 \mu \mathrm{M}$, absolute intensity threshold $=1560$, objects area $>50 \mu \mathrm{M}^{2}$ ) for each dataset. Quantification of total surface area and sphericity was performed using Imaris. Measurements were exported and statistically assessed using Prism 9.0 (Graphpad).

For GFP fluorescence intensity quantification, the GFP signal intensity of 30 random cells in the ONT+ and ONT- retina per fish $(\mathrm{N}=6)$ was collected using Fiji ImageJ and the corrected total cell fluorescence (CTCF) was obtained (after L. Hammond, 2014, Measuring cell fluorescence using ImageJ, The University of Queensland, Australia, https://theolb.readthedocs.io/en/latest/imaging/measuring-cell-fluorescence-using-

imagej.html). Briefly, using the freehand tool in ImageJ, single RGCs were outlined as a region of interest (ROI) and GFP intensity was measured as was as the fluorescence of the background for every retina. CTCF was then calculated as follows: Integrated Density - (Area of selected cell X Mean fluorescence of background readings). Relative intensity was calculated as the ratio of the CTCF in ONT+ RGCs divided by that of ONT- RGCs.

Localization of pSTAT3 expression to RGCs was confirmed with surface creation in Imaris 9.6.0. Quantification of pSTAT3 levels was performed as described (Osborne et al. 2018) using FIJI. Briefly, is/2b:GFP labeling was used to identify the location and depth of the ganglion cell layer (GCL). RGC volumes were converted to Z-projections and background subtraction and speckle removal was performed on all images. Threshold 
226 levels for pSTAT3 were determined from ONT- samples and consistent thresholds were

227 then applied to all images in ONT+ samples and integrated density was measured. The

228 expression level of pSTAT3 was calculated as the ratio of the average integrated density 229 of pSTAT3 in 1dpi, 7dpi, P6/1dpi, or P6/7dpi retinae to the average integrated density of 230 the contralateral ONT- eye.

231

232 Statistics

233 All statistical analyses were performed using Prism 9.0 (Graphpad). Data were presented 234 as mean+/-SD, with the exception of cleaved caspase-3 images which show mean+/235 SEM. For multiple comparisons, Kruskal-Wallis ANOVA followed by Dunn's multiple 236 comparisons tests between groups were performed. For comparisons between two 237 groups, non-parametric Mann-Whitney tests were performed, with the exception of 238 sphericity comparison in which an unpaired t-test with Welch's correction was performed. 239 P-values, sample sizes, and statistical analyses for each experiment are included in the 240 figure legends. 


\section{RESULTS AND DISCUSSION:}

\section{Zebrafish retain the majority of RGCs after ONT}

To enable isolation of RGCs after injury, we first verified that GFP remained expressed in RGCs of adult is/2b:GFP fish (Pittman et al., 2008). In retinal cryosections, is/2b:GFP cells co-labeled with DAPI and the RGC-specific marker, zn-8 (Larison and Bremiller, 1990), and $64.86 \pm 8.44 \%$ of cells within the GCL were is/2b:GFP+ (Fig. 1A).

To create an ON injury, we performed optic nerve transection (ONT). Injured retinae from the left eye (ONT+) and sham surgery retinae from the right eye (ONT-) were collected $1,3,7$, or 14 days post injury (dpi) (Fig. 1B). We confirmed that there were no differences in cell number between the uninjured left and right eye (S. Chen; data not shown). To quantify RGC survival after ONT, we counted is/2b:GFP ${ }^{+}$RGCs from eight regions (four in the peripheral retina and four in the central retina (Fig. 1B)), then divided counts from the ONT+ retina by those from the ONT- retina of the same fish. RGC survival was $94.44 \pm 5.45 \%$ at $1 \mathrm{dpi}$ and $92.52 \pm 3.54 \%$ at $3 \mathrm{dpi}$; however, survival decreased to $76.35 \pm 2.58 \%$ at $7 \mathrm{dpi}(\mathrm{p}=0.0009$ ). At $14 \mathrm{dpi}, \mathrm{RGC}$ numbers recovered to $98.71 \pm 4.82 \%$ (Fig. $1 C, D)$.

is/2b:GFP intensity in ONT+ RGCs declined by $40.43 \pm 9.99 \%$ at $7 \mathrm{dpi}$ relative to ONT- RGCs ( $p<0.0001)$, raising the possibility that GFP intensity was falling below the detection threshold after ONT due to compromised health and not death of is/2b:GFP ${ }^{+}$ RGCs. Cleaved caspase 3 immunostaining revealed that $12.44 \pm 1.79 \%$ of RGCs in the ONT+ retinae were caspase $3^{+}$at $7 \mathrm{dpi}$, compared to $0.06 \pm 0.01 \%$ of RGCs in the ONT- 
265 retina (Fig. S1). Moreover, no BrdU ${ }^{+}$cells were detected in the GCL of ONT+ retinae at 7

266 dpi, indicating that RGCs had not yet regenerated (S. Chen, data not shown). Taken

267 together, these data indicate that, despite transient reduction in is/2b:GFP levels and

268 limited death, most is/2b:GFP ${ }^{+}$RGCs are indeed preserved in zebrafish after ONT.

\section{Identification of neuroprotective factors and pathways after ONT}

To identify neuroprotective signals/pathways in RGCs, is/2b:GFP+ RGCs were

272 FACS-isolated from ONT+ and ONT- retinae at 24hpi and utilized for RNA-seq (Fig 2A).

273 We identified 308 differentially expressed genes (DEGs) (Fig. 2B), of which 56 were

274 upregulated and 252 were downregulated (Tables S1,2). We reasoned that

275 neuroprotective factors would be upregulated upon ONT, and the upregulated DEG group

276 included stat3, irf9, sox11b, lepr, and socs $3 b$, which encode components/regulators of

277 the JAK/STAT signaling pathway (Fig. 2C; (Villarino et al., 2015)), in addition to other

278 neuroprotective and pro-regenerative genes such as gap43 (Chung et al., 2020), atf3

279 (Kole et al., 2020), and atoh7 (Brodie-Kommit et al., 2021). Moreover, the interferon regulatory factor genes, irf9 and irf1b (Langevin et al., 2013), and the chemokine receptor, cxcr4b (García-Cuesta et al., 2019), were also upregulated, suggesting activation of

282 innate immune responses in RGCs after ONT. Pathway enrichment analyses indicated that JAK/STAT signaling was the most highly enriched pathway in RGCs after ONT (Fig. 2D). Furthermore, DEGs associated with the adipocytokine signaling pathway, which

286 ONT (Fig. 2D). Downregulated DEGs revealed that neuroactive ligand receptor 
287 interactions, MAPK, and calcium signaling pathways were all significantly changed after

288 ONT (Fig. 2D).

289

290

291

292

293

294

295

296

297

298

299

300

301

302

303

304

305

306

307

308

309

310

We also investigated gene expression changes at $12 \mathrm{hpi}$. Using the same selection criteria as for $24 \mathrm{hpi}$ analyses yielded limited numbers of DEGs at $12 \mathrm{hpi}$, with 5 upregulated and 3 downregulated (Table S3). Amongst these upregulated DEGs, however, was the leukocyte recruitment cytokine, cxcl12b (García-Cuesta et al., 2019), whose receptor, $c x c r 4 b$, was upregulated at $24 \mathrm{hpi}$. Relaxing our selection criteria from an FDR $p$-value $<0.05$ to a $p$-value $<0.05$ revealed 49 upregulated and 31 downregulated DEGs (Tables S4,5). stat3 and socs3b were amongst the upregulated group, as was the pro-inflammatory cytokine, il1b (Hasegawa et al., 2017), further suggesting activation of the JAK/STAT and innate immune pathways in RGCs after ONT.

\section{JAKISTAT pathway activity is required for RGC survival after ONT}

JAK/STAT activity contributes to RGC survival in multiple injury contexts (Boyd et al., 2003; Huang et al., 2007; Luo et al., 2007) and facilitates ON and retinal regeneration (Elsaeidi et al., 2014; Kassen et al., 2009; Leibinger et al., 2013; Mehta et al., 2016; Park et al., 2004; Todd et al., 2016; Zhao et al., 2014). To confirm JAK/STAT pathway activation after ONT, we assessed phosphorylated-STAT3 (pSTAT3) levels in ONT+ and ONT- retinae (Fig. 3A; Movie S1). pSTAT3 levels were significantly increased in ONT+ RGCs (Fig. 3B; $p<0.01$ ), supporting the notion that Stat3 may be neuroprotective after ONT. To determine whether JAK/STAT pathway activity is required for RGC survival after ONT, we performed intravitreal (IV) injections of a pan-Jak inhibitor, Pyridone 6 (P6), or $0.05 \%$ DMSO at the time of ONT and again at 1dpi (Fig. 3C). P6-injection resulted in 
311 significant reductions to pSTAT3 levels in ONT+ RGCs at 1dpi and 7dpi, supporting the

312 efficacy of P6 in blocking Jak activation in zebrafish (Fig. 3D,E). We next quantified

313 is/2b:GFP ${ }^{+}$RGC survival at 7dpi when Jak activity was impaired (Fig. 3F,G). DMSO had

314 no effect on RGC survival, nor did P6 alone ( $p=0.4796$; Fig. 3G). However, IV P6 injection

315 in conjunction with ONT resulted in a significant reduction in RGC survival to $39.44 \pm 6.99 \%$

$316(p<0.0001)$. These data demonstrate a role for JAKISTAT pathway activity in protecting

317 zebrafish RGCs after ONT.

\section{Innate immune response involvement after ONT}

After ONI, the effects of injury-activated immune responses are varied. In some

Tezel, 2019; Mac Nair et al., 2016; Nadal-Nicolás et al., 2017; Williams et al., 2017), while

in others, the stimulation of limited neuroinflammation induces pro-survival and

332 facilitate repair and regeneration (Leach et al., 2020; White et al., 2017). In zebrafish, the 
334 (Craig et al., 2008). Consistent with other reports (Mitchell et al., 2018), 4C4+ macrophages/microglia were located throughout the ONT- retina, including in the GCL (Fig. 4A,B; Movie S2). At 1dpi, the number of $4 \mathrm{C}^{+}$cells appeared to increase in the $\mathrm{GCL}$ 1dpi (Fig. 4C). Due to morphology and close proximity in the ONT retinae, it was difficult to identify single macrophages/microglia for counting and therefore we quantified the percent area covered by mCherry ${ }^{+}$cells within the GCL (Fig. 4D). When compared to ONT- retinae, mCherry ${ }^{+}$cells covered nearly 6 times more GCL area after ONT $(p=0.0006 ;$ Fig. 4D). Macrophage/microglia morphologies change upon activation,

whereby quiescent cells with a ramified morphology take on a more spherical/amoeboid

(Fig. 4B,C), and quantification of $\mathrm{mCherry}^{+}$cell sphericity revealed a significant increase at $1 \mathrm{dpi}$ (Fig. 4E; $p<0.0001$ ); collectively, showing that macrophages/microglia accumulate in the GCL and become activated after ONT.

\section{Blocking inflammation or depletion of macrophages/microglia protects RGCs after}

Pro-inflammatory cytokines, interferon response factors, and leukocyte 354 recruitment factors were upregulated in is/2b:GFP ${ }^{+}$RGCs post-ONT (Fig. 2C; Tables 355 S1,S3,S4) and macrophages/microglia accumulate and become activated in the ONT+ 356 GCL (Fig.4 A-D). These data suggest that macrophage/microglia-mediated inflammation 
357 might contribute to RGC death after ONT, in opposition to JAK/STAT-mediated

358 neuroprotection. To test this hypothesis, we inhibited inflammation after ONT via

359 dexamethasone treatment, a strategy shown to protect RGCs in other injury models

360 (Bollaerts et al., 2019; Dutt et al., 2010; Gallina et al., 2015; Jovanovic et al., 2020), and

361 quantified RGC survival. Experimentally, $2 \mu \mathrm{L}$ of $100 \mu \mathrm{M}$ dexamethasone or $0.05 \%$ DMSO

362 (vehicle control) was IV injected into is/2b:GFP fish at the time of ONT and again at $1 \mathrm{dpi}$

363 and tissue was collected at 7dpi (after Fig. 3C). Dexamethasone alone had no effect on

364 is/2b:GFP ${ }^{+}$RGC survival in the ONT- retina $(96.56 \pm 8.67 \%, p=0.5998)$, but significantly

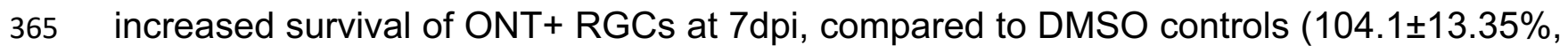

$366 \mathrm{p}<0.05$; Fig. 4G). Dexamethasone-mediated inhibition of inflammation prevents the

367 recruitment of macrophages/microglia in some contexts (Tsarouchas et al., 2018; White

368 et al., 2017), but not others (Chatzopoulou et al., 2016; Warchol, 1999; Xie et al., 2019).

369 Quantification of the percent of the GCL covered by mpeg1:mCherry ${ }^{+}$

370 macrophages/microglia in DMSO- and dexamethasone-injected retinae revealed no

371 significant differences after ONT (Fig. 4H, p=0.0623). Taken together, these data

372 demonstrate that impairment of inflammation after ONT rescued RGC survival but didn't

373 suppress the recruitment of macrophages/microglia to the GCL.

Previous reports have identified roles for microglia in contributing to RGC death after a variety of insults (Bosco et al., 2008; Jovanovic et al., 2020; Takeda et al., 2018).

377 However, other studies have shown that microglia are dispensable for RGC survival after 378 ON crush (Hilla et al., 2017), and may instead provide neuroprotective and/or pro379 regenerative signals (Bell et al., 2018; Sappington et al., 2006). To directly test the 
requirement of macrophages/microglia in modulating RGC death in zebrafish after ONT, we depleted macrophages/microglia using PLX3397, a potent inhibitor of the colonystimulating factor 1 receptor (CSF1R). CSF1R activity is required for macrophage/microglia differentiation (Lin et al., 2008; Sherr et al., 1985) and PLX3397 has been utilized effectively in zebrafish (e.g. Conedera et al., 2019; Leach et al., 2020; Van Dyck et al., 2021). Animals were immersed in 500nM PLX3397 one day prior to ONT and retinae were collected at $7 \mathrm{dpi}$. To validate the efficiency of macrophage/microglia depletion by PLX3397, we quantified the percent area of the GCL occupied by mpeg 1:mCherry ${ }^{+}$macrophages/microglia. PLX3397 significantly reduced the coverage of mpeg 1:mCherry ${ }^{+}$cells in both the ONT- and ONT+ GCL (Fig. 4I,J). Similar to dexamethasone-mediated RGC protection (Fig. 4F,G), PLX3397-mediated depletion of macrophages/microglia also rescued RGC survival at 7dpi $(103.49 \pm 12.01 \%$, $p<0.05$; Fig. $4 \mathrm{I}, \mathrm{K})$. Finally, we determined whether the neuroprotective effects mediated by JAK/STAT activity in RGCs after ONT were required in the absence of macrophages/microglia. PLX3397-mediated depletion of macrophages/microglia also rescued RGC survival at 7dpi after JAK/STAT pathway inhibition using P6 (107.60 $\pm 9.32 \%$, p<0.05; Figs. 4K,S2), indicating that JAK/STAT activity is dispensable in the absence of macrophage/microglia recruitment. neurotoxic signals emanating from macrophages/microglia and JAK/STAT pathway activation in zebrafish RGCs regulates their survival after ONI. As noted above, Stat3 upregulation has been associated with RGC survival in some experimental contexts 
403 (Huang et al., 2007; Luo et al., 2007). Despite this, overall RGC survival is limited under 404 physiological conditions, with over $90 \%$ of RGCs lost within 28 days after injury in mice 405 (Li et al., 2020). This is not true for all mammals, however. Indeed, the naked mole-rat 406 retains many RGCs after injury, to at least 28 days (Park et al., 2017). Interestingly, while pStat3 is nearly absent in mouse RGCs, even after injury, it increases significantly in

408

409

410

411

412

413

414

415

416

417

418

419

420

421

422

423

424

425

mole-rat RGCs after ON crush, supporting a possible role for Stat3 activity in RGC neuroprotection.

Counter to our expectations, pSTAT3 localization was predominantly cytoplasmic in ONT+ RGCs, rather than nuclear (Fig. 3A, Movie S1). Interestingly, this observation is consistent with cytoplasmic pSTAT3 localization in the regenerating zebrafish retina (Elsaeidi et al., 2014) and in mouse motor neurons responding to cytokine stimulation (Selvaraj et al., 2012). Stat3 possesses transcription-independent functions such as cytoplasmically regulating autophagy (Shen et al., 2012). Stat3 also localizes to mitochondria after cytokine stimulation in mouse RGCs where it regulates metabolic functions and enhances axon regeneration after ONI (Luo et al., 2016). Thus, it is possible that the function of Stat3 in RGC neuroprotection could be transcription-independent.

Not all zebrafish RGCs survive ONT and caspase $3^{+}$RGCs appeared to be a nonrandom pattern in the ONT+ retina (Fig. S1). This may indicate that there are RGC subtype(s) that are more susceptible to ONI, similar to what has been observed in mice (Tran et al., 2019). RGC subtypes in zebrafish have been recently characterized (Kölsch et al., 2021) and additional studies will be required to determine if specific subtypes are 
426 lost after ONT, and if so, whether these subtypes lack the ability to upregulate JAK/STAT

427 activity after injury. Finally, it will be of interest to identify the signals emanating from

428 macrophages/microglia that activate death and/or pro-survival pathways in zebrafish

429 RGCs after injury, as these would also be promising targets around which neuroprotective

430 therapies for glaucoma could be developed (García-Bermúdez et al., 2021; Rashid et al.,

$4312019)$. 


\section{ACKNOWLEDGEMENTS:}

433

434 The work described herein was supported by a grant from the BrightFocus Foundation

435 National Glaucoma Research Program (G2020277) to JMG; an unrestricted grant from

436 Xiangya Hospital of Central South University and China Scholar Council for studying in

437 Pittsburgh to SC; NIH CORE Grant P30-EY08098 to the Department of Ophthalmology;

438 the Eye \& Ear Foundation of Pittsburgh, and an unrestricted grant from Research to

439 Prevent Blindness, New York, NY. We're grateful to Dick Barrett and Ben Carr for

440 technical assistance and to Dr. Hugh Hammer for expert zebrafish husbandry. 


\section{LITERATURE CITED}

Almasieh, M., Wilson, A. M., Morquette, B., Cueva Vargas, J. L. and Di Polo, A. (2012). The molecular basis of retinal ganglion cell death in glaucoma. Prog. Retin. Eye Res. 31, 152-181.

Bariş, M. and Tezel, G. (2019). Immunomodulation as a Neuroprotective Strategy for Glaucoma Treatment. Curr Ophthalmol Rep 7, 160-169.

Bell, K., Und Hohenstein-Blaul, N. von T., Teister, J. and Grus, F. (2018). Modulation of the Immune System for the Treatment of Glaucoma. Curr. Neuropharmacol. 16, 942-958.

Bollaerts, I., Van Houcke, J., Beckers, A., Lemmens, K., Vanhunsel, S., De Groef, L. and Moons, L. (2019). Prior Exposure to Immunosuppressors Sensitizes Retinal Microglia and Accelerates Optic Nerve Regeneration in Zebrafish. Mediators Inflamm. 2019 6135795. https://doi.org/10.1155/2019/6135795

Bosco, A., Inman, D. M., Steele, M. R., Wu, G., Soto, I., Marsh-Armstrong, N., Hubbard, W. C., Calkins, D. J., Horner, P. J. and Vetter, M. L. (2008). Reduced retina microglial activation and improved optic nerve integrity with minocycline treatment in the DBA/2J mouse model of glaucoma. Invest. Ophthalmol. Vis. Sci. 49, 1437-1446.

Boyd, Z. S., Kriatchko, A., Yang, J., Agarwal, N., Wax, M. B. and Patil, R. V. (2003). Interleukin-10 Receptor Signaling through STAT-3 Regulates the Apoptosis of Retinal Ganglion Cells in Response to Stress. Investigative Ophthalmology \& Visual Science 44, 5206-5211.

Brodie-Kommit, J., Clark, B. S., Shi, Q., Shiau, F., Kim, D. W., Langel, J., Sheely, C., Ruzycki, P. A., Fries, M., Javed, A., et al. (2021). Atoh7-independent specification of retinal ganglion cell identity. Sci Adv 7, 11 eabe4983.

Chang, E. E. and Goldberg, J. L. (2012). Glaucoma 2.0: neuroprotection, neuroregeneration, neuroenhancement. Ophthalmology 119, 979-986.

Chatzopoulou, A., Heijmans, J. P. M., Burgerhout, E., Oskam, N., Spaink, H. P., Meijer, A. H. and Schaaf, M. J. M. (2016). Glucocorticoid-Induced Attenuation of the Inflammatory Response in Zebrafish. Endocrinology 157, 2772-2784.

Chung, D., Shum, A. and Caraveo, G. (2020). GAP-43 and BASP1 in Axon Regeneration: Implications for the Treatment of Neurodegenerative Diseases. Front Cell Dev Biol 8, doi: 10.3389/fcell.2020.567537.

Cigliola, V., Becker, C. J. and Poss, K. D. (2020). Building bridges, not walls: spinal cord regeneration in zebrafish. Dis. Model. Mech. 13, doi: 10.1242/dmm.044131

Conedera, F. M., Pousa, A. M. Q., Mercader, N., Tschopp, M. and Enzmann, V. 
(2019). Retinal microglia signaling affects Müller cell behavior in the zebrafish following laser injury induction. Glia 67, 1150-1166.

Craig, S. E. L., Calinescu, A.-A. and Hitchcock, P. F. (2008). Identification of the molecular signatures integral to regenerating photoreceptors in the retina of the zebrafish. J. Ocul. Biol. Dis. Infor. 1, 73-84.

Dhara, S. P., Rau, A., Flister, M. J., Recka, N. M., Laiosa, M. D., Auer, P. L. and Udvadia, A. J. (2019). Cellular reprogramming for successful CNS axon regeneration is driven by a temporally changing cast of transcription factors. Sci. Rep. 9, 14198.doi: 10.1038/s41598-019-50485-6.

Diekmann, H., Kalbhen, P. and Fischer, D. (2015). Characterization of optic nerve regeneration using transgenic zebrafish. Front. Cell. Neurosci. 9, 118.doi: 10.3389/fncel.2015.00118.

Dutt, M., Tabuena, P., Ventura, E., Rostami, A. and Shindler, K. S. (2010). Timing of corticosteroid therapy is critical to prevent retinal ganglion cell loss in experimental optic neuritis. Investigative Ophthalmology \& Visual Science 51, 1439-1445.

Ellett, F., Pase, L., Hayman, J. W., Andrianopoulos, A. and Lieschke, G. J. (2011). mpeg1 promoter transgenes direct macrophage-lineage expression in zebrafish. Blood 117, e49-56.

Elsaeidi, F., Bemben, M. A., Zhao, X.-F. and Goldman, D. (2014). Jak/Stat signaling stimulates zebrafish optic nerve regeneration and overcomes the inhibitory actions of Socs3 and Sfpq. J. Neurosci. 34, 2632-2644.

Fausett, B. V. and Goldman, D. (2006). A Role for a1 Tubulin-Expressing Müller Glia in Regeneration of the Injured Zebrafish Retina. J. Neurosci. 26, 6303-6313.

Gallina, D., Zelinka, C. P., Cebulla, C. M. and Fischer, A. J. (2015). Activation of glucocorticoid receptors in Müller glia is protective to retinal neurons and suppresses microglial reactivity. Exp. Neurol. 273, 114-125.

García-Bermúdez, M. Y., Freude, K. K., Mouhammad, Z. A., van Wijngaarden, P., Martin, K. K. and Kolko, M. (2021). Glial Cells in Glaucoma: Friends, Foes, and Potential Therapeutic Targets. Front. Neurol. 12, doi: 10.3389/fneur.2021.624983.

García-Cuesta, E. M., Santiago, C. A., Vallejo-Díaz, J., Juarranz, Y., RodríguezFrade, J. M. and Mellado, M. (2019). The Role of the CXCL12/CXCR4/ACKR3 Axis in Autoimmune Diseases. Front. Endocrinol. 10, doi: 10.3389/fendo.2019.00585.

Hasegawa, T., Hall, C. J., Crosier, P. S., Abe, G., Kawakami, K., Kudo, A. and Kawakami, A. (2017). Transient inflammatory response mediated by interleukin-1 $\beta$ is required for proper regeneration in zebrafish fin fold. Elife $\mathbf{6}$, https://doi.org/10.7554/eLife.22716. 
Hilla, A. M., Diekmann, H. and Fischer, D. (2017). Microglia Are Irrelevant for Neuronal Degeneration and Axon Regeneration after Acute Injury. J. Neurosci. 37, 6113-6124.

Huang, Y., Cen, L.-P., Choy, K. W., van Rooijen, N., Wang, N., Pang, C. P. and Cui, Q. (2007). JAK/STAT pathway mediates retinal ganglion cell survival after acute ocular hypertension but not under normal conditions. Exp. Eye Res. 85, 684-695.

Huang, T., Li, H., Zhang, S., Liu, F., Wang, D. and Xu, J. (2021). Nrn1 Overexpression Attenuates Retinal Ganglion Cell Apoptosis, Promotes Axonal Regeneration, and Improves Visual Function Following Optic Nerve Crush in Rats. Journal of Molecular Neuroscience 71, 66-79.

Jovanovic, J., Liu, X., Kokona, D., Zinkernagel, M. S. and Ebneter, A. (2020). Inhibition of inflammatory cells delays retinal degeneration in experimental retinal vein occlusion in mice. Glia 68, 574-588.

Kadye, R., Stoffels, M., Fanucci, S., Mbanxa, S. and Prinsloo, E. (2020). A STAT3 of Addiction: Adipose Tissue, Adipocytokine Signalling and STAT3 as Mediators of Metabolic Remodelling in the Tumour Microenvironment. Cells 9,1043. doi:10.3390/cells9041043.

Kanagaraj, P., Chen, J. Y., Skaggs, K., Qadeer, Y., Conner, M., Cutler, N., Richmond, J., Kommidi, V., Poles, A., Affrunti, D., et al. (2020). Microglia Stimulate Zebrafish Brain Repair Via a Specific Inflammatory Cascade. Cold Spring Harbor Laboratory 2020.10.08.330662.

Karlstetter, M., Scholz, R., Rutar, M., Wong, W. T., Provis, J. M. and Langmann, T. (2015). Retinal microglia: just bystander or target for therapy? Prog. Retin. Eye Res. 45, 30-57.

Kassen, S. C., Thummel, R., Campochiaro, L. A., Harding, M. J., Bennett, N. A. and Hyde, D. R. (2009). CNTF induces photoreceptor neuroprotection and Müller glial cell proliferation through two different signaling pathways in the adult zebrafish retina. Exp. Eye Res. 88, 1051-1064.

Kole, C., Brommer, B., Nakaya, N., Sengupta, M., Bonet-Ponce, L., Zhao, T., Wang, C., Li, W., He, Z. and Tomarev, S. (2020). Activating Transcription Factor 3 (ATF3) Protects Retinal Ganglion Cells and Promotes Functional Preservation After Optic Nerve Crush. Invest. Ophthalmol. Vis. Sci. 61, 31. doi:10.1167/iovs.61.2.31

Kölsch, Y., Hahn, J., Sappington, A., Stemmer, M., Fernandes, A. M., Helmbrecht, T. O., Lele, S., Butrus, S., Laurell, E., Arnold-Ammer, I., et al. (2021). Molecular classification of zebrafish retinal ganglion cells links genes to cell types to behavior. Neuron 109, 645-662.e9.

Lahne, M., Nagashima, M., Hyde, D. R. and Hitchcock, P. F. (2020). Reprogramming Müller Glia to Regenerate Retinal Neurons. Annu Rev Vis Sci 6, 171-193. 
Langevin, C., Aleksejeva, E., Passoni, G., Palha, N., Levraud, J.-P. and Boudinot, P. (2013). The antiviral innate immune response in fish: evolution and conservation of the IFN system. J. Mol. Biol. 425, 4904-4920.

Larison, K. D. and Bremiller, R. (1990). Early onset of phenotype and cell patterning in the embryonic zebrafish retina. Development 109, 567-576.

Leach, L. L., Hanovice, N. J., George, S. M., Gabriel, A. E. and Gross, J. M. (2020). The immune response is a critical regulator of zebrafish retinal pigment epithelium regeneration. biorxiv 2020.08.14.250043.

Leibinger, M., Andreadaki, A., Diekmann, H. and Fischer, D. (2013). Neuronal STAT3 activation is essential for CNTF- and inflammatory stimulation-induced CNS axon regeneration. Cell Death \& Disease 4, e805-e805.

Li, L., Huang, H., Fang, F., Liu, L., Sun, Y. and Hu, Y. (2020). Longitudinal Morphological and Functional Assessment of RGC Neurodegeneration After Optic Nerve Crush in Mouse. Front. Cell. Neurosci. 14, 109. doi:10.3389/fncel.2020.00109

Lin, H., Lee, E., Hestir, K., Leo, C., Huang, M., Bosch, E., Halenbeck, R., Wu, G., Zhou, A., Behrens, D., et al. (2008). Discovery of a cytokine and its receptor by functional screening of the extracellular proteome. Science 320, 807-811.

Luo, J.-M., Cen, L.-P., Zhang, X.-M., Chiang, S. W.-Y., Huang, Y., Lin, D., Fan, Y.-M., Van Rooijen, N., Lam, D. S. C., Pang, C. P., et al. (2007). PI3K/akt, JAK/STAT and MEK/ERK pathway inhibition protects retinal ganglion cells via different mechanisms after optic nerve injury. European Journal of Neuroscience 26, 828842.

Luo, X., Ribeiro, M., Bray, E. R., Lee, D.-H., Yungher, B. J., Mehta, S. T., Thakor, K. A., Diaz, F., Lee, J. K., Moraes, C. T., et al. (2016). Enhanced Transcriptional Activity and Mitochondrial Localization of STAT3 Co-induce Axon Regrowth in the Adult Central Nervous System. Cell Rep. 15, 398-410.

Mac Nair, C. E., Schlamp, C. L., Montgomery, A. D., Shestopalov, V. I. and Nickells, R. W. (2016). Retinal glial responses to optic nerve crush are attenuated in Bax-deficient mice and modulated by purinergic signaling pathways. $J$. Neuroinflammation 13, 93. https://doi.org/10.1186/s12974-016-0558-y

Mehta, S. T., Luo, X., Park, K. K., Bixby, J. L. and Lemmon, V. P. (2016). Hyperactivated Stat3 boosts axon regeneration in the CNS. Exp. Neurol. 280, 115120.

Mitchell, D. M., Lovel, A. G. and Stenkamp, D. L. (2018). Dynamic changes in microglial and macrophage characteristics during degeneration and regeneration of the zebrafish retina. J. Neuroinflammation 15, 163. https://doi.org/10.1186/s12974018-1185-6 
Nadal-Nicolás, F. M., Jiménez-López, M., Salinas-Navarro, M., Sobrado-Calvo, P., Vidal-Sanz, M. and Agudo-Barriuso, M. (2017). Microglial dynamics after axotomy-induced retinal ganglion cell death. J. Neuroinflammation 14, 218. https://doi.org/10.1186/s12974-017-0982-7

Osborne, A., Khatib, T. Z., Songra, L., Barber, A. C., Hall, K., Kong, G. Y. X., Widdowson, P. S. and Martin, K. R. (2018). Neuroprotection of retinal ganglion cells by a novel gene therapy construct that achieves sustained enhancement of brain-derived neurotrophic factor/tropomyosin-related kinase receptor-B signaling. Cell Death Dis. 9, 1007. https://doi.org/10.1038/s41419-018-1041-8

Park, K. K., Luo, X., Mooney, S. J., Yungher, B. J., Belin, S., Wang, C., Holmes, M. M. and He, Z. (2017). Retinal ganglion cell survival and axon regeneration after optic nerve injury in naked mole-rats. J. Comp. Neurol. 525, 380-388.

Park, K., Luo, J.-M., Hisheh, S., Harvey, A. R. and Cui, Q. (2004). Cellular mechanisms associated with spontaneous and ciliary neurotrophic factor-cAMPinduced survival and axonal regeneration of adult retinal ganglion cells. J. Neurosci. 24, 10806-10815.

Pereiro, X., Ruzafa, N., Acera, A., Fonollosa, A., Rodriguez, F. D. and Vecino, E. (2018). Dexamethasone protects retinal ganglion cells but not Müller glia against hyperglycemia in vitro. PLoS One 13, e0207913.

Pittman, A. J., Law, M.-Y. and Chien, C.-B. (2008). Pathfinding in a large vertebrate axon tract: isotypic interactions guide retinotectal axons at multiple choice points. Development 135, 2865-2871.

Rashid, K., Akhtar-Schaefer, I. and Langmann, T. (2019). Microglia in Retinal Degeneration. Front. Immunol. 10, 1975. doi: 10.3389/fimmu.2019.01975.

Sappington, R. M., Chan, M. and Calkins, D. J. (2006). Interleukin-6 protects retinal ganglion cells from pressure-induced death. Invest. Ophthalmol. Vis. Sci. 47, 29322942.

Selvaraj, B. T., Frank, N., Bender, F. L. P., Asan, E. and Sendtner, M. (2012). Local axonal function of STAT3 rescues axon degeneration in the pmn model of motoneuron disease. J. Cell Biol. 199, 437-451.

Shen, S., Niso-Santano, M., Adjemian, S., Takehara, T., Malik, S. A., Minoux, H., Souquere, S., Mariño, G., Lachkar, S., Senovilla, L., et al. (2012). Cytoplasmic STAT3 represses autophagy by inhibiting PKR activity. Mol. Cell 48, 667-680.

Sherr, C. J., Rettenmier, C. W., Sacca, R., Roussel, M. F., Look, A. T. and Stanley, E. R. (1985). The c-fms proto-oncogene product is related to the receptor for the mononuclear phagocyte growth factor, CSF-1. Cell 41, 665-676.

Syc-Mazurek, S. B. and Libby, R. T. (2019). Axon injury signaling and 
compartmentalized injury response in glaucoma. Prog. Retin. Eye Res. 73, 100769. doi:10.1016/j.preteyeres.2019.07.002

Takeda, A., Shinozaki, Y., Kashiwagi, K., Ohno, N., eto, K., Wake, H., Nabekura, J. and Koizumi, S. (2018). Microglia mediate non-cell-autonomous cell death of retinal ganglion cells. Glia 66, 2366-2384.

Todd, L., Squires, N., Suarez, L. and Fischer, A. J. (2016). Jak/Stat signaling regulates the proliferation and neurogenic potential of Müller glia-derived progenitor cells in the avian retina. Sci. Rep. 6, 35703. https://doi.org/10.1038/srep35703

Todd, L., Palazzo, I., Suarez, L., Liu, X., Volkov, L., Hoang, T. V., Campbell, W. A., Blackshaw, S., Quan, N. and Fischer, A. J. (2019). Reactive microglia and IL1 $\beta /$ IL-1R1-signaling mediate neuroprotection in excitotoxin-damaged mouse retina. J. Neuroinflammation 16, 118. https://doi.org/10.1186/s12974-019-1505-5

Tran, N. M., Shekhar, K., Whitney, I. E., Jacobi, A., Benhar, I., Hong, G., Yan, W., Adiconis, X., Arnold, M. E., Lee, J. M., et al. (2019). Single-Cell Profiles of Retinal Ganglion Cells Differing in Resilience to Injury Reveal Neuroprotective Genes. Neuron 104, 1039-1055.e12.

Tsarouchas, T. M., Wehner, D., Cavone, L., Munir, T., Keatinge, M., Lambertus, M., Underhill, A., Barrett, T., Kassapis, E., Ogryzko, N., et al. (2018). Dynamic control of proinflammatory cytokines II- $1 \beta$ and Tnf- $\alpha$ by macrophages in zebrafish spinal cord regeneration. Nature Communications 9,4670 https://doi.org/10.1038/s41467-018-07036-w.

Uribe, R. A. and Gross, J. M. (2007). Immunohistochemistry on cryosections from embryonic and adult zebrafish eyes. CSH Protoc. 2007, db.prot4779.

Van Dyck, A., Bollaerts, I., Beckers, A., Vanhunsel, S., Glorian, N., van Houcke, J., van Ham, T. J., De Groef, L., Andries, L. and Moons, L. (2021). Müller gliamyeloid cell crosstalk accelerates optic nerve regeneration in the adult zebrafish. Glia. doi:10.1002/glia.23972

Villarino, A. V., Kanno, Y., Ferdinand, J. R. and O'Shea, J. J. (2015). Mechanisms of Jak/STAT signaling in immunity and disease. J. Immunol. 194, 21-27.

Warchol, M. E. (1999). Immune cytokines and dexamethasone influence sensory regeneration in the avian vestibular periphery. J. Neurocytol. 28, 889-900.

White, D. T., Sengupta, S., Saxena, M. T., Xu, Q., Hanes, J., Ding, D., Ji, H. and Mumm, J. S. (2017). Immunomodulation-accelerated neuronal regeneration following selective rod photoreceptor cell ablation in the zebrafish retina. Proc. Natl. Acad. Sci. U. S. A. 114, E3719-E3728.

Williams, P. A., Marsh-Armstrong, N., Howell, G. R. and Lasker/IRRF Initiative on Astrocytes and Glaucomatous Neurodegeneration Participants (2017). 
Neuroinflammation in glaucoma: A new opportunity. Exp. Eye Res. 157, 20-27.

665

666

667

668

669

670

671

672

673

674

675

676

677

Xie, Y., Tolmeijer, S., Oskam, J. M., Tonkens, T., Meijer, A. H. and Schaaf, M. J. M. (2019). Glucocorticoids inhibit macrophage differentiation towards a proinflammatory phenotype upon wounding without affecting their migration. Dis. Model. Mech. 12, dmm037887. doi: 10.1242/dmm.037887.

Zhang, C., Li, H., Liu, M.-G., Kawasaki, A., Fu, X.-Y., Barnstable, C. J. and ShaoMin Zhang, S. (2008). STAT3 activation protects retinal ganglion cell layer neurons in response to stress. Exp. Eye Res. 86, 991-997.

Zhao, X.-F., Wan, J., Powell, C., Ramachandran, R., Myers, M. G., Jr and Goldman, D. (2014). Leptin and IL-6 family cytokines synergize to stimulate Müller glia reprogramming and retina regeneration. Cell Rep. 9, 272-284.

Zou, S., Tian, C., Ge, S. and Hu, B. (2013). Neurogenesis of retinal ganglion cells is not essential to visual functional recovery after optic nerve injury in adult zebrafish. PLoS One 8, e57280. 
A

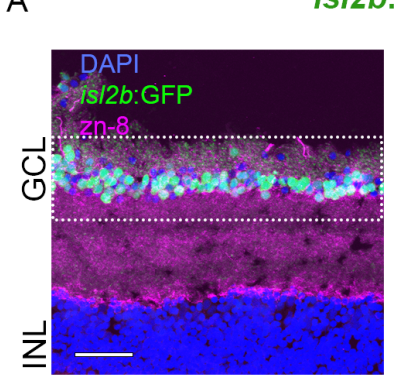

is/2b:GFP+
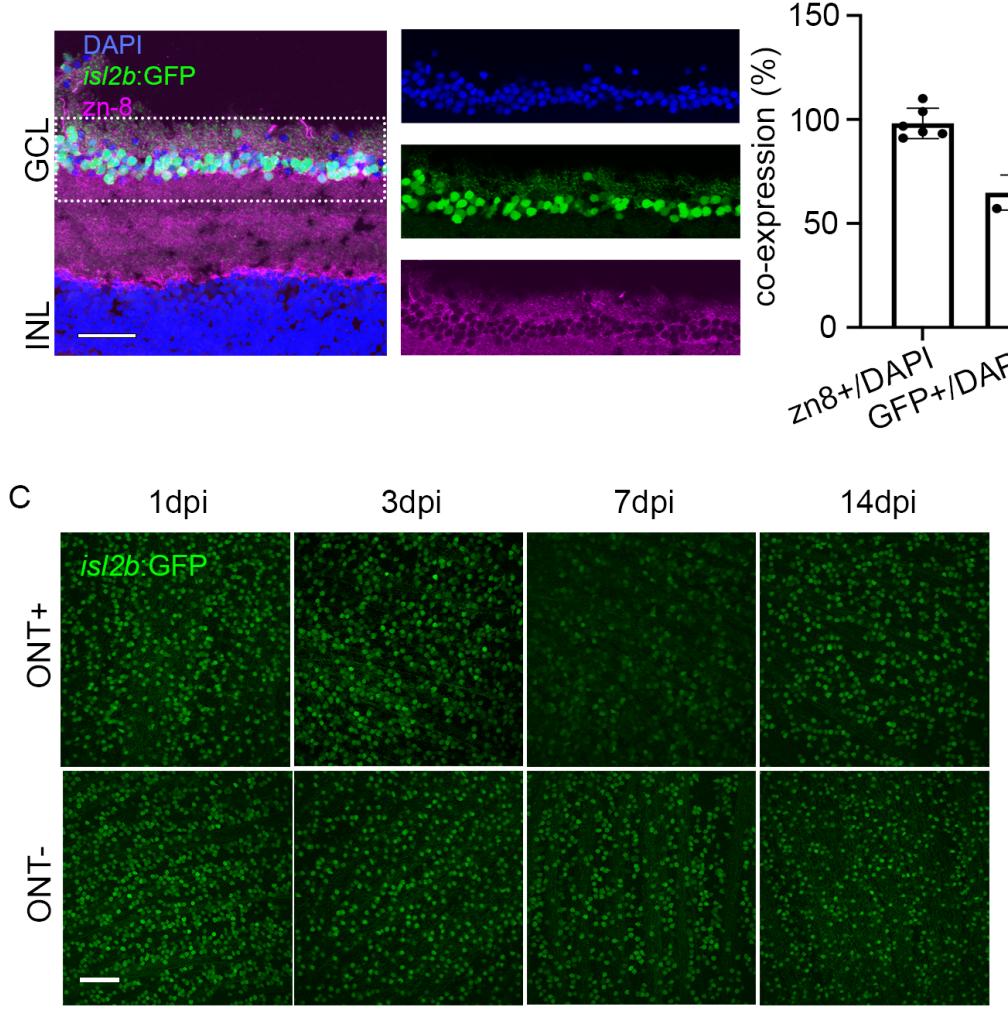

B
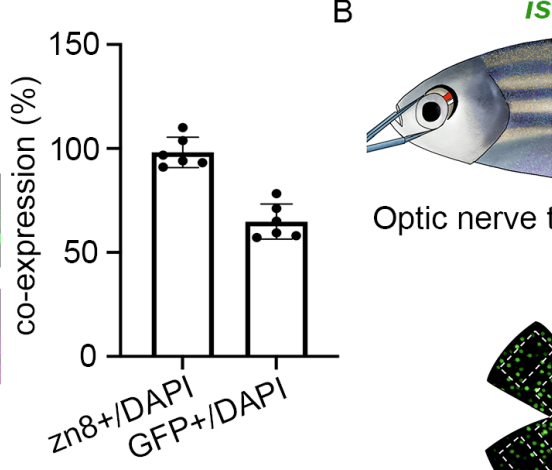

is/ $2 b: G F P+$

Optic nerve transection(ONT)

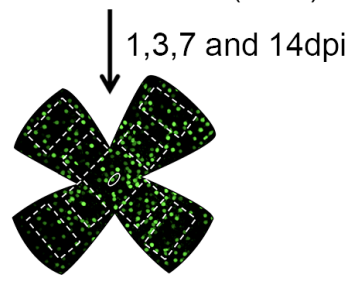

Average of RGCs in all regions

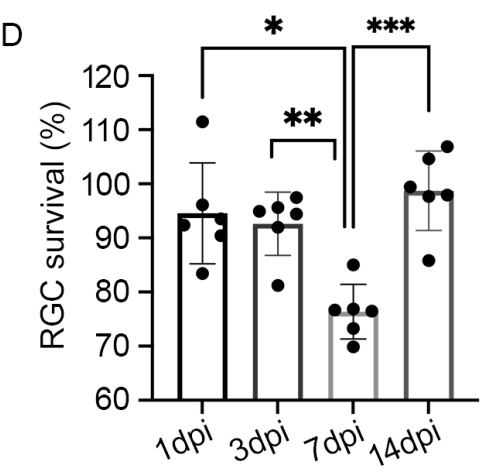

\section{Figure1: Zebrafish RGCs are preserved after ONT}

(A) Immunolabeling of RGCs in the ganglion cell layer (GCL) with zn-8 (magenta) in the adult is/2b:GFP (green) retinae. $\sim 65 \%$ of DAPI (blue) stained RGCs were is/2b:GFP+. (B) Overview of optic nerve transection (ONT) and RGC survival analyses. (C) Images of 1, 3, 7, and 14dpi flat-mount retinae. (D) RGC survival percentages at 1, 3, 7, and $14 \mathrm{dpi}\left(\mathrm{n}=6 /\right.$ day). Shown are mean $\pm S D$; ${ }^{*} \mathrm{p}<0.05$; ${ }^{* *} \mathrm{p}<0.01$; ${ }^{* * *} \mathrm{p}<0.001$; Kruskal Wallis ANOVA w/ Dunn's multiple comparisons. Scale bars $=50 \mu \mathrm{m}$. 
A is/2b:GFP+

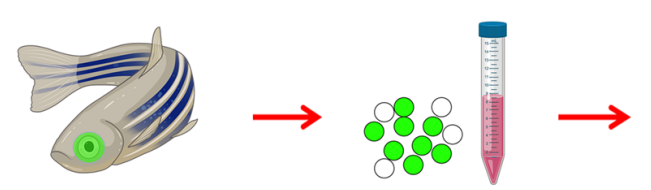

Collect ONT+ and ONT- Single cell suspension retina samples at $24 \mathrm{hpi}$

B

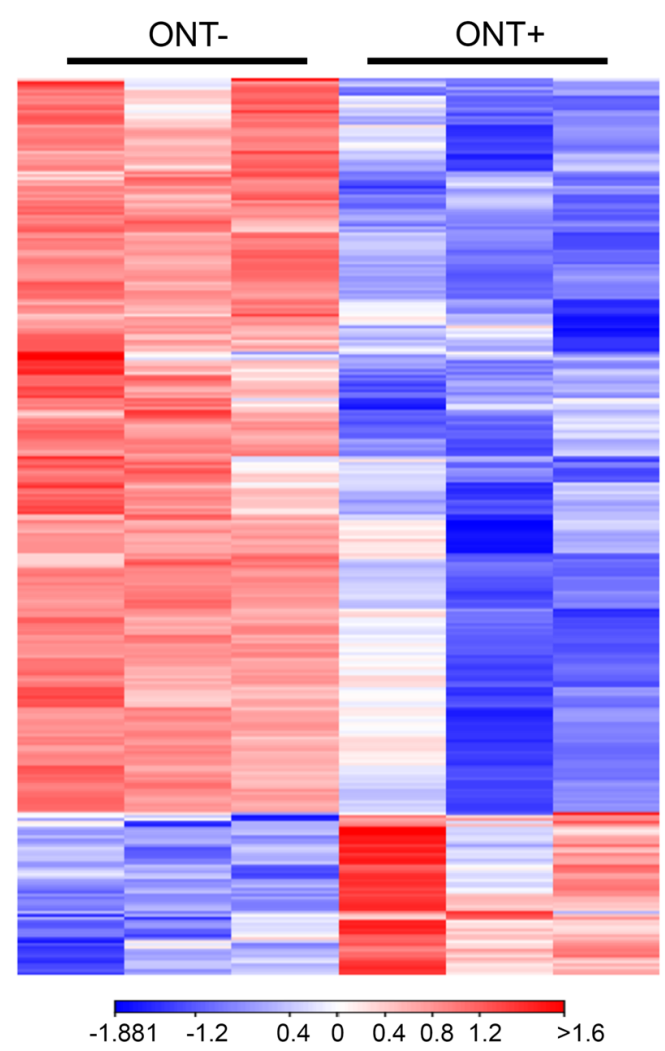

C

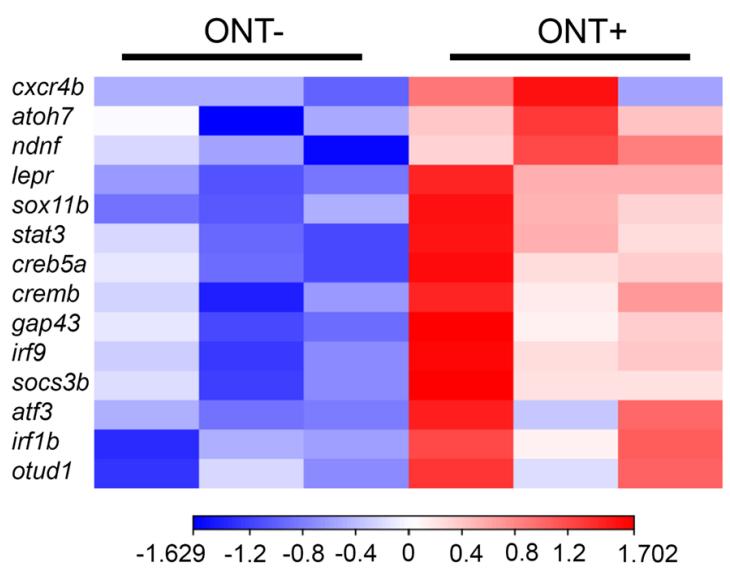

D

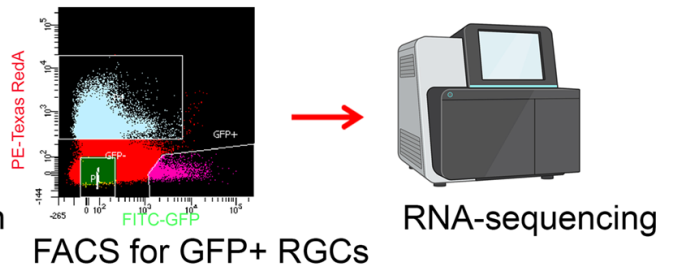

FACS for GFP+ RGCs

RNA-sequencing

KEGG Pathway Analysis
Down

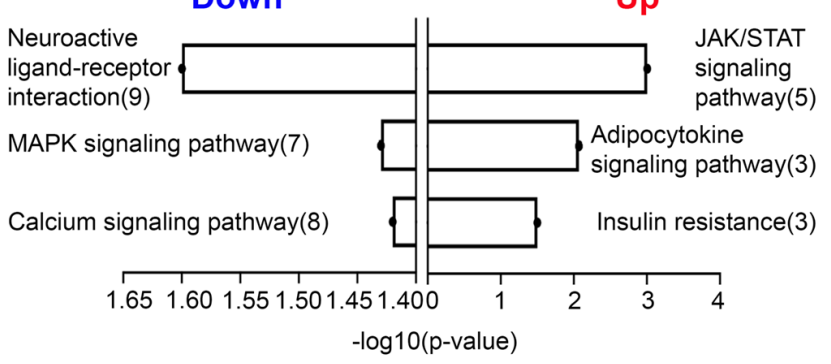

Figure 2: Identification of differentially expressed genes in is/2b:GFP+RGs after ONT.

(A) Experimental workflow for FACS-isolation of is/2b:GFP+ RGCs. An example FACS plot showing a cell sorting gate is included. Icons were adapted from BioRender.com. (B) Heatmap showing hierarchical clustering of 308 DEGs at $24 \mathrm{hpi}$ from three biological replicates. (C) Heatmap highlighting DEGs of interest based on known neuroprotective and pro-regenerative functions. Heatmap legends show $\log _{2}$ TPM. (D) Pathway enrichment analysis using the KEGG database showing top-3 down- and up-regulated pathways after ONT. 
A

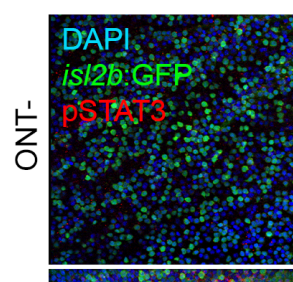

$\stackrel{+}{\mathrm{s}}$

c

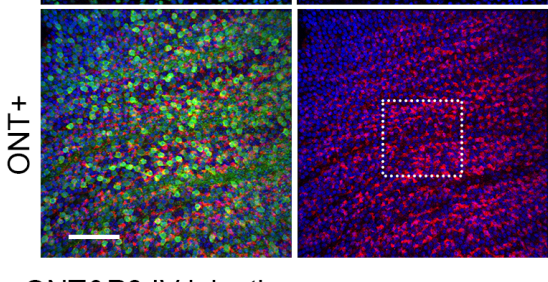

ONT\&P6 IV injection

P6 IV injection
B
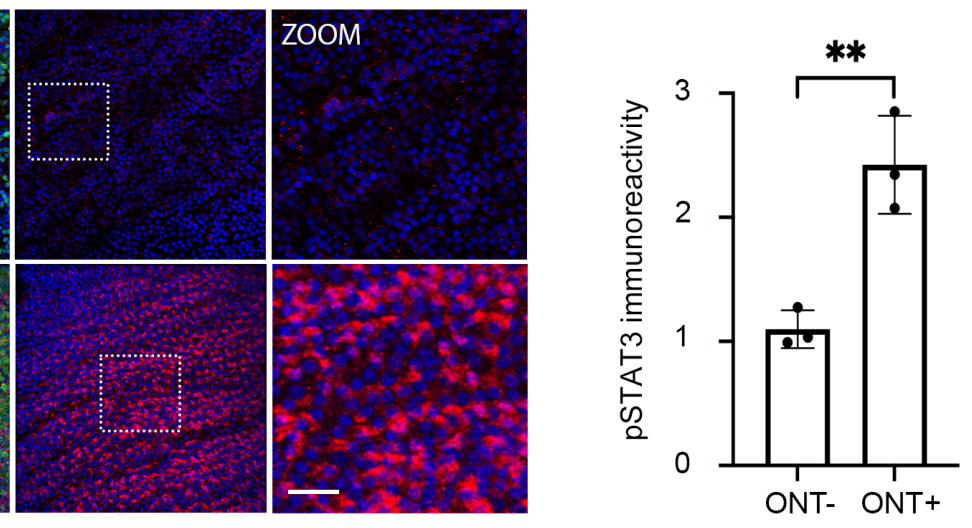

Sample collection

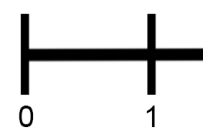

D

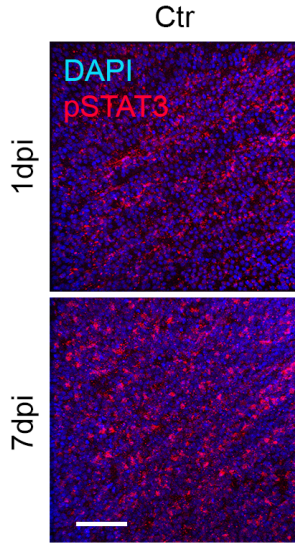

F

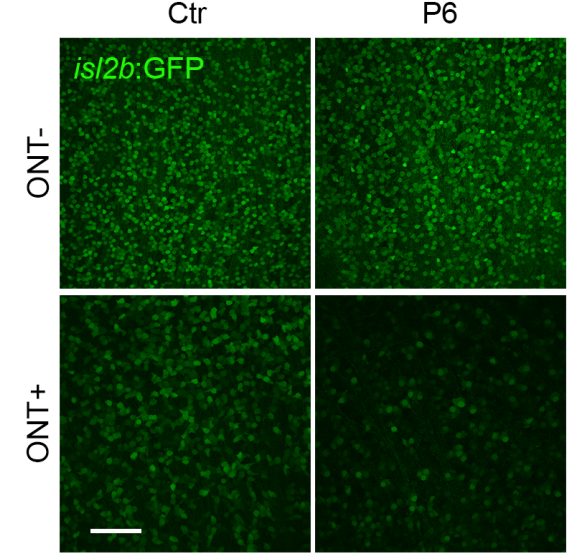

P6

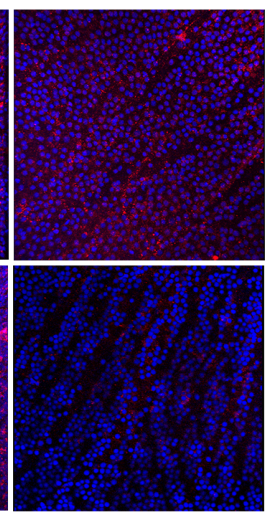

G

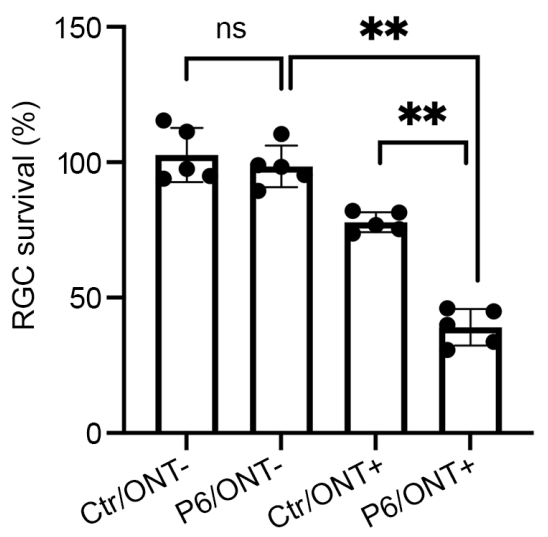




\section{Figure 3: JAK/STAT pathway activity is required for RGC survival after ONT}

(A) pSTAT3 expression (red) in flat-mount is/2b:GFP ONT- and ONT+ retinae at $1 \mathrm{dpi}$. Nuclei stained with DAPI (blue). Boxed regions are $3 x$ zooms of $40 x$ images. (B) Quantification of pSTAT3 levels at 1dpi. pSTAT3 levels in ONT+ RGCs relative to levels normalized to those in ONT- RGCs. Shown are mean $\pm S D$ of $n=3$ for each group; ${ }^{* *} p<0.01$; Mann-Whitney test. (C) Experimental paradigm to assess Jak requirement during RGC survival after ONT. (D) pSTAT3 expression in ONT+ is/2b:GFP retinal flat mounts at 1 and 7dpi +/- intravitreal (IV) injection of the Jak inhibitor, P6. DMSO was used as control (Ctr). (E) Quantification of pSTAT3 expression after P6 application at 1 and $7 \mathrm{dpi}$. $\mathrm{N}=3$ /condition. Shown are mean $\pm \mathrm{SD}$; ${ }^{*} \mathrm{p}<0.05$, Mann-Whitney test. (F) Images of 7dpi P6- or DMSO-injected flat-mount is/2b:GFP retinae. (G) Quantification of $R G C$ survival after P6 injection ( $n=5 /$ condition). Shown are mean $\pm S D$; ${ }^{* *} p<0.01$; Kruskal Wallis ANOVA with Dunn's multiple comparisons. Scale bars $=50 \mu \mathrm{m}$ and $150 \mu \mathrm{m}$ on zoomed images in A. 
A

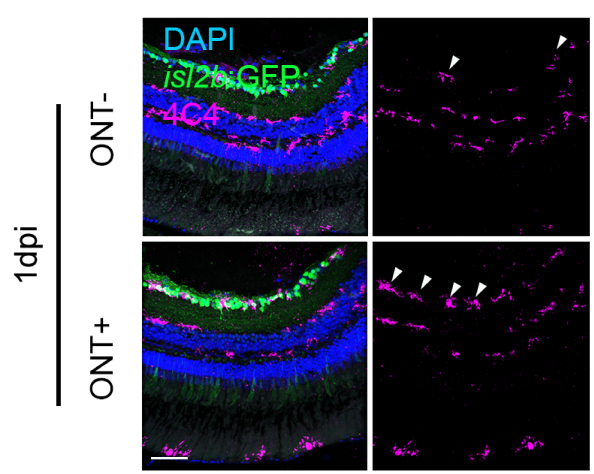

C

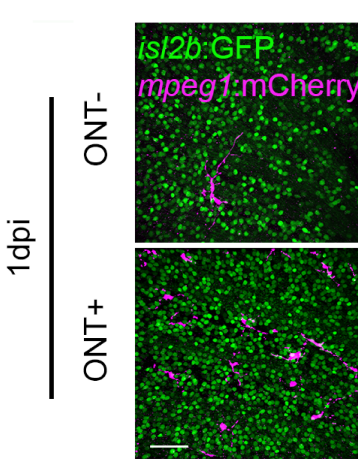

F
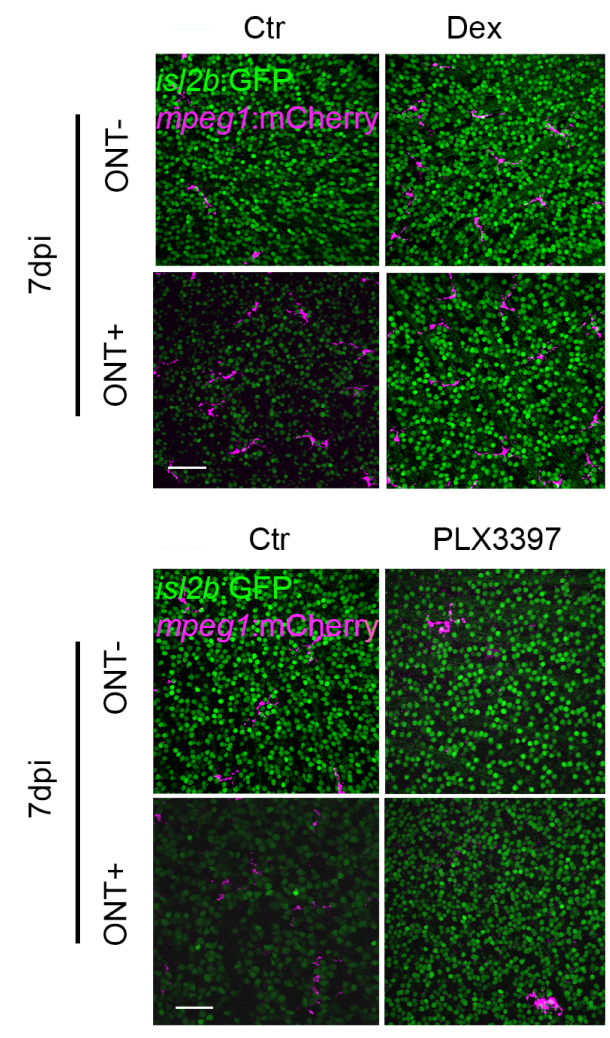

B

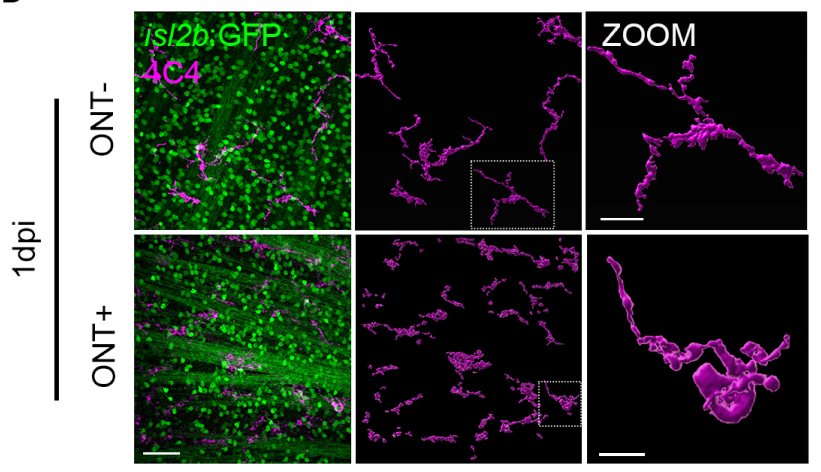

$\mathrm{E}$
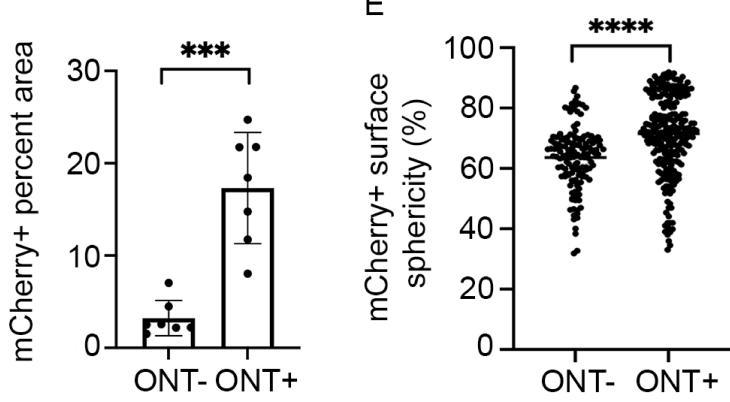

H
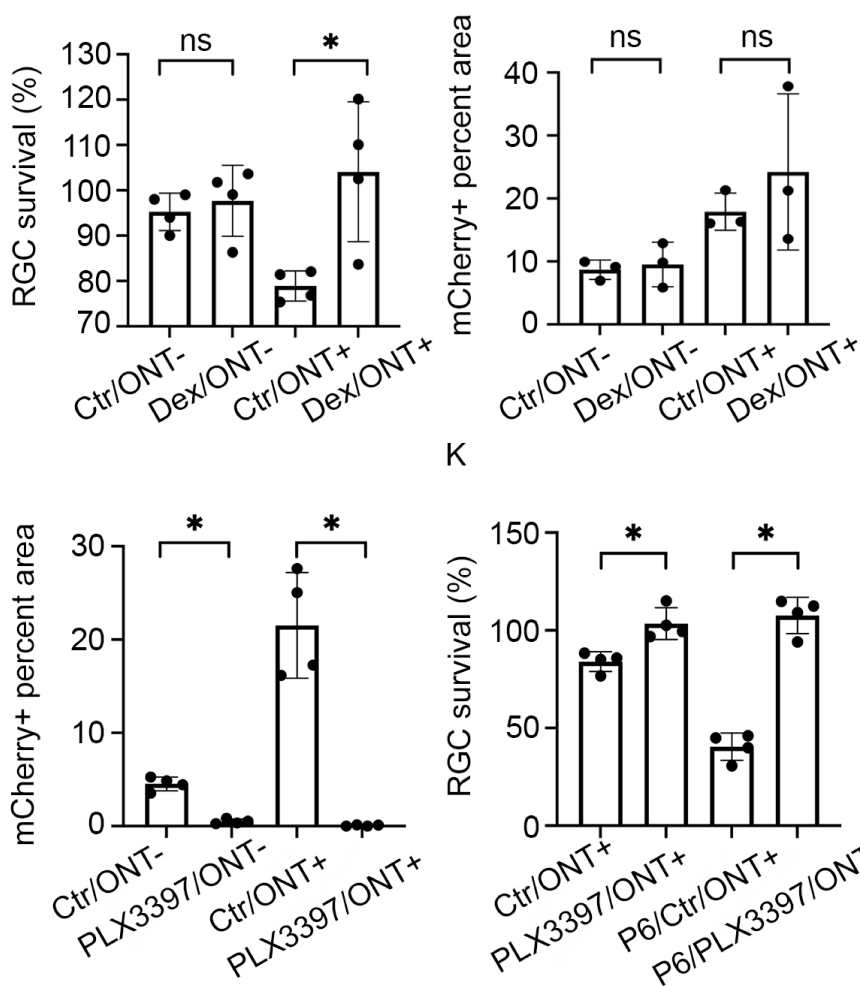


\section{Figure 4: Macrophages/microglia are recruited to the GCL after ONT and mediate RGC death}

Immunostaining of 4C4 (magenta) on is/2b:GFP retinal cryosections (A) and retinal flat mounts at $1 \mathrm{dpi}$ with Imaris surface renderings of $4 \mathrm{C}^{+}$macrophages/microglia (B). (C) Images of $1 \mathrm{dpi}$ retinal flat-mounts from is/2b:GFP;mpeg1:mCherry animals; macrophages/microglia (magenta). (D) Quantification of the GCL surface area occupied by $\mathrm{mCherry}^{+}$macrophages/microglia at $1 \mathrm{dpi}(\mathrm{n}=4 /$ condition). Shown are mean $\pm S D$; ${ }^{* * *} p<0.001$; Mann-Whitney test. (E) Violin plot showing a significant increase in sphericity of mCherry+ macrophages/microglia in ONT+ retinae compared to ONTcontrols ( $n=140$ in ONT- and $n=272$ in ONT+). ${ }^{* * * *} p<0.0001$; unpaired t-test with Welch's correction. (F) Flat-mount images of is/2b:GFP;mpeg1:mCherry retinae after intravitreal injection of dexamethasone (Dex) or DMSO (Ctr) at 7dpi. (G) RGC survival in dexamethasone-treated retinae increased significantly at $7 \mathrm{dpi}$ when compared to control ( $n=4 /$ condition). Shown are mean $\pm S D$; ${ }^{*} p<0.05$; Kruskal Wallis ANOVA test with Dunn's multiple comparisons. (H) Quantification of mCherry ${ }^{+}$macrophage/microglia coverage of the GCL after ONT and dexamethasone or DMSO injection ( $n=3 /$ condition). Shown are mean $\pm S D$; Kruskal Wallis ANOVA test with Dunn's multiple comparisons. No significant differences were detected. (I) Flat-mount images of is/2b:GFP;mpeg1:mCherry retinae after PLX3397 or control treatment (Ctr) at 7dpi. (J) Quantification of mCherry ${ }^{+}$macrophage/microglia coverage of the GCL after ONT and PLX3397 treatment ( $n=4 /$ condition). Shown are mean $\pm S D$; ${ }^{*} p<0.05$; Kruskal Wallis ANOVA test with Dunn's multiple comparisons. (K) RGC survival in PLX3397-treated retinae increased significantly at $7 \mathrm{dpi}$ when compared to control. Similarly, RGC survival in PLX3397-treated retinae increased significantly after P6 addition over DMSO controls ( $n=4 /$ condition). Shown are mean $\pm S D$; ${ }^{*} P<0.05$; Mann-Whitney test. Scale bars $=$ $50 \mu \mathrm{m}$. 